\title{
ON THE DISSIPATIVE EVOLUTION EQUATIONS ASSOCIATED WITH THE ZAKHAROV-SHABAT SYSTEM WITH A QUADRATIC SPECTRAL PARAMETER
}

\author{
JYH-HAO LEE
}

\begin{abstract}
In this paper we derive some results for the Zakharov-Shabat system of the form $d m / d x=z^{2}[J, m]+(z Q+P) m ; J$ is diagonal and skewHermitian $[8,10,12]$. Following the idea of R. Beals and R. R. Coifman, we estimate the wedge products of the columns of $m$ by $L^{2}$-norm of the potential $(Q, P)$ [4]. By this result we have the global existence of the dissipative evolution equations associated with this spectral problem if the generic initial data $(Q(x, 0), P(x, 0))=\left(Q_{0}, P_{0}\right)$ is of Schwartz class.
\end{abstract}

\section{DiRect AND INVERSE SCATTERING FOR THE Z-S SYSTEM WITH A QUADRATIC PARAMETER}

By a potential here we mean a pair of functions $(Q, P): \mathbf{R} \rightarrow M_{n}(\mathbf{C})=$ set of $n \times n$ complex matrices; $Q$ is off-diagonal and the diagonal part of $P$ equals the diagonal part of $Q(\operatorname{ad} J)^{-1} Q$, and $Q, Q_{x}, P, P_{x} \in L^{1}$. We consider the following spectral problem: Given $z \notin \Sigma=\left\{z: \operatorname{Im}\left(z^{2}\right)=0\right\}$, find $m(\cdot, z): R \rightarrow M_{n}(\mathbf{C})$ with

$$
\begin{aligned}
& \partial m(z, z) / \partial x=z^{2}[J, m(x, z)]+(z Q+P) m(x, z), \text { where } \\
& J=\operatorname{diag}\left(i d_{1}, i d_{2}, \ldots, i d_{n}\right), d_{1}<d_{2}<d_{3}<\cdots<d_{n} ; \\
& m(\cdot, z) \text { bounded, } m(x, z) \rightarrow I \text { as } x \rightarrow-\infty .
\end{aligned}
$$

Let

$$
\Omega_{+}=\left\{z: \operatorname{Im}\left(z^{2}\right)>0\right\}, \quad \Omega_{-}=\left\{z: \operatorname{Im}\left(z^{2}\right)<0\right\} .
$$

For a certain set of potentials, called generic, $m$ has the following properties $[8,10]$ :

(1.3) For any $z \in \Sigma$, there is a unique matrix $v(z)$ such that for all $x, m^{+}(x, z)=m^{-}(x, z) \exp \left(x z^{2} J\right) v(z) \exp \left(-x z^{2} J\right)$, where $m^{ \pm}(x, z)=$ limit of $m$ on $\Sigma$ from $\Omega_{ \pm}$;

Received by the editors May 4, 1988.

1980 Mathematics Subject Classification (1985 Revision). Primary 35Q20, 35 Q15.

Key words and phrases. Integrable evolution equations, inverse scattering transform, ZakharovShabat system. 
$m(x, \cdot)$ has a finite number of poles at $D=\left\{z_{1}, z_{2}, \ldots, z_{N}\right\}$ (which do not depend on $x$ ); for any $z_{j}$, there is a matrix $v\left(z_{j}\right)$ such that

$\operatorname{Res}\left(m(x, \cdot), z_{j}\right)=\lim _{z \rightarrow z_{j}} m(x, z) \exp \left(x z_{j}^{2} J\right) v\left(z_{j}\right) \exp \left(-x z_{j}^{2} J\right) ;$

The $\operatorname{map}(Q, P) \rightarrow v=\left\{v(z) ; z_{1}, z_{2}, \ldots, z_{N}, v\left(z_{1}\right), v\left(z_{2},\right)\right.$, $\left.\ldots, v\left(z_{N}\right)\right\}$ is injective. We denote this map by $\mathrm{sd}$;

i.e., $\operatorname{sd}(Q, P)=v$.

By a similar argument in [2], we can show that generic Schwartz class potentials form an open and dense set in Schwartz class potentials. To make our exposition clearer, from now on we assume $(Q, P)$ is of Schwartz class. If $(Q, P)$ is generic, we call the associated function $v: \Sigma \cup D \rightarrow M_{n}(\mathbf{C})$ the scattering data. We introduce some more notation. For any matrix $A$, we let $d_{k}^{+}(A)$ and $d_{k}^{-}(A)$ denote the upper and lower $k \times k$ principal minors. The scattering data satisfies the following constraints:

$d_{k}^{+}(v)=1,1 \leq k \leq n ; d_{k}^{-}(v) \neq 0,1 \leq k \leq n$.

(1.7) If $z_{j}$ is a pole, then $v\left(z_{j}\right)=c_{j} e_{k, k+1}$ for some $k$ if $z_{j} \in \Omega_{+} ; v\left(z_{j}\right)=c_{j} e_{k+1, k}$ for some $k$ if $z_{j} \in \Omega_{-}$.

(1.8) The winding number of $d_{k}^{-}(v)=\beta_{k}^{+}-\beta_{k}^{-}$, where $\beta_{k}^{+}$is the number of $z_{j}$ in $\Omega_{+}$such that the $n-k+1$ column of $v\left(z_{j}\right) \neq 0$ and $\beta_{k}^{-}$is the number of $z_{j}$ in $\Omega_{-}$such that the $n-k$ column of $v\left(z_{j}\right) \neq 0$; i.e., $\beta_{k}^{+}-\beta_{k}^{-}=\int_{\Sigma} d\left[\arg d_{k}^{-}(v)\right]$ and $\Sigma$ is oriented such that $\Omega_{+}$is in the left side. $v(z)-I$ is of Schwartz class in $\Sigma$.

Let $\mathrm{SD}=$ set of $v$ satisfying (1.6)-(1.9). Given $v \in \mathrm{SD}$, then the inverse problem amounts to solving an analytic factorization problem (Riemann-Hilbert problem) with one parameter $x$. The set $v \in \mathrm{SD}$ such that $m$ is solvable is open and dense in $\mathrm{SD}$, and such $v$ is called generic $[2,8,12]$. Note that $v \in \mathrm{SD}$ is generic if and only if there exists a potential $(Q, P)$ such that $\operatorname{sd}(Q, P)=v$.

More precisely, the inverse problem is solved following the argument of Beals and Coifman in the case of the first-order system [2]. For $x \leq 0$ the solution $m$ has the form $m=r m^{\#} \exp \left(x z^{2} J\right) u \exp \left(-x z^{2} J\right)$, where $u$ is a piecewise rational function, $m^{\#}$ satisfies an equation of the form (1.1) with $v^{\#}$ and $v^{\#}-1$ small, and $r$ is rational in $z$. The solution for $x \geq 0$ has the same form with different $u, m^{\#}, r$. Given $v \in \mathrm{SD}$, the function $u$ and $m^{\#}$ above can be determined. Determination of $r$ amounts to solving a finite system of linear algebraic equations with parameter $x$, and $v=\operatorname{sd}(Q, P)$ if and only if these equations are solvable for all $x \in R$. 


\section{Estimate of $m$ IN $L^{2}$-NORM OF $(Q, P)$}

Recall that the spectral problem is of the form

$$
\begin{aligned}
& d m / d x=z^{2}[J, m]+(z Q+p) m, \quad z \notin \Sigma, \\
& m(x, z) \rightarrow I, \quad \text { as } x \rightarrow \infty, \quad P^{\mathrm{diag}}=\left(Q(\operatorname{ad} J)^{-1} Q\right)^{\mathrm{diag}} .
\end{aligned}
$$

In Lee's dissertation a technique of integration by parts enables one to control the term $z Q$ for large $z$ [8]. An alternative method is given in [9] to control the $z$-dependence. We may solve $m(x, z)$ for $|z| \leq N$ by the argument of Beals and Coifman [2]. In (2.0) the constraint on the diagonal part of $P$ enables us to pose the inverse problem easily. (This constraint implies $\lim _{|z| \rightarrow \infty} m(x, z)=I$, so in the inverse probem we may solve $m$ normalized at $z=\infty$.) For technical reasons, we need the following transformation:

Let $\tilde{m}=\exp \left(-\int_{-\infty}^{x}\left(Q(\operatorname{ad} J)^{-1} Q\right)^{\mathrm{diag}}\right) m$; then $\tilde{m}$ satisfies

$$
d \tilde{m} / d x=z^{2}[J, \tilde{m}]+z \tilde{Q} m+\tilde{P} \widetilde{m}, \quad z \notin \Sigma, \tilde{m}(x, z) \rightarrow I \text { as }
$$$$
x \rightarrow \infty \text { where }
$$

$$
\begin{aligned}
& \widetilde{Q}=\exp \left(-\int_{-\infty}^{x}\left(Q(\operatorname{ad} J)^{-1} Q\right)^{\mathrm{diag}}\right) Q \exp \left(\int_{-\infty}^{x}\left(Q(\operatorname{ad} J)^{-1} Q\right)^{\mathrm{diag}}\right), \\
& \widetilde{P}=\exp \left(-\int_{-\infty}^{x}\left(Q(\operatorname{ad} J)^{-1} Q\right)^{\mathrm{diag}}\right) P^{\mathrm{off}} \exp \left(\int_{-\infty}^{x}\left(Q(\operatorname{ad} J)^{-1} Q\right)^{\mathrm{diag}}\right) .
\end{aligned}
$$

Here $\widetilde{Q}$ and $\widetilde{P}$ are both off-diagonal. Note that

$$
\begin{gathered}
\left(Q(\operatorname{ad} J)^{-1} Q\right)^{\operatorname{diag}}=\left(\widetilde{Q}(\operatorname{ad} J)^{-1} \widetilde{Q}\right)^{\operatorname{diag}}, \\
\|\widetilde{Q}\|_{L^{2}} \leq \exp \left(c\|Q\|_{L^{2}}\right)\|Q\|_{L^{2}}, \quad\|\widetilde{P}\|_{L^{2}} \leq \exp \left(c\|Q\|_{L^{2}}\right)\|P\|_{L^{2}}, \\
\left|m_{i j}(x, z)\right| \leq\left|\tilde{m}_{i j}(x, z)\right| \exp \left(c\|Q\|_{L^{2}}\right), \\
\left|\lim _{x \rightarrow \infty} \tilde{m}_{i j}(x, z)\right| \leq \exp \left(\|Q\|_{L^{2}}\right)\left|\lim _{x \rightarrow \infty} m_{i j}(x, z)\right|, \quad \text { where }\|Q\|_{L^{2}} \equiv \sum_{i, j}\left\|Q_{i j}\right\|_{L^{2}} .
\end{gathered}
$$

If $Q^{*}=-Q$, then $\left(Q(\operatorname{ad} J)^{-1} Q\right)^{\text {diag }}$ is purely imaginary and $\left|\tilde{m}_{i j}(x, z)\right|=$ $\left|m_{i j}(x, z)\right|$. From now on we write $m, Q, P$ instead of $\tilde{m}, \widetilde{Q}, \widetilde{P}$. We may convert (2.1) into an integral equation

$$
\begin{aligned}
& \frac{d m_{k, l}(x, z)}{d x} \\
& \quad=\left\{\begin{array}{l}
\delta_{k l}+\int_{-\infty}^{x} e^{(x-y) z^{2} i\left(d_{k}-d_{l}\right)}[(z Q(y)+P(y)) m(x, z)]_{k l} d y \quad \text { for } k \geq l, \\
-\int_{x}^{\infty} e^{(x-y) z^{2} i\left(d_{k}-d_{l}\right)}[(z Q(y)+P(y)) m(y, z)]_{k l} d y \text { for } k<l .
\end{array}\right.
\end{aligned}
$$

The first column $m_{1}$ of $m$ satisfies a Volterra equation. But the remaining columns $m_{j}$ of $m$ satisfy Fredholm equations. Following the idea of Beals 
and Coifman, the wedge products $m_{1} \wedge m_{2}, m_{1} \wedge m_{2} \wedge m_{3}, \ldots, m_{1} \wedge m_{2} \wedge \cdots \wedge$ $m_{n-1}$ satisfy Volterra equations. If we normalize $m$ at $x=\infty$, then $m_{n}$, $m_{n} \wedge m_{n-1}, m_{n} \wedge m_{n-1} \wedge m_{n-2}, \ldots$ also satisfy Volterra equations.

(2.3) Lemma. If $a \in M_{n}(\mathbf{C})$ is an invertible matrix with columns $a_{1}, a_{2}, \ldots$, $a_{n}$, then $a$ is uniquely determined by the wedge products

$$
\begin{aligned}
& a_{1}, a_{1} \wedge a_{2}, a_{1} \wedge a_{2} \wedge a_{3}, \ldots, a_{1} \wedge a_{2} \wedge \cdots \wedge a_{n}, \\
& a_{n}, a_{n-1} \wedge a_{n}, a_{n-2} \wedge a_{n-1} \wedge a_{n}, \ldots, a_{1} \wedge a_{2} \wedge \cdots \wedge a_{n} .
\end{aligned}
$$

Proof. See Beals and Coifman [2].

(2.4) Lemma. Given $Q=\left(Q_{i j}\right)_{n \times n}, \quad Q_{i i}=0, \quad Q_{i j} \in L^{2}(R),\|Q\|_{L^{2}} \equiv$ $\sum_{i m j}\left\|Q_{i j}\right\|_{L^{2}}, \quad c_{1}=0, \quad c_{i} \in \mathbf{C}, \operatorname{Re} c_{i}^{2}<0$, and $\left|c_{i}^{2}\right| \leq K\left|\operatorname{Re} c_{i}^{2}\right|, \quad i \geq 2$, then there are unique absolutely continuous bounded functions $u_{i}$ satisfying the following ordinary differential equations:

$$
\left\{\begin{array}{l}
d u_{1} / d x=\sum_{j \neq 1} Q_{1 j} u_{j}, \\
d u_{i} / d x=c_{i}^{2} u_{i}+\left(c_{i}^{2} Q_{i 1} u_{1}+\sum_{j \neq 1, i} c_{i} Q_{i j} u_{j}\right), \quad i=2,3, \ldots, n, \\
u_{1}(-\infty)=1, \quad u_{i}(-\infty)=0, \quad i \geq 2,
\end{array}\right.
$$

and $\left\|u_{1}\right\|_{L^{\infty}} \leq C=C\left(K,\|Q\|_{L^{2}}\right),\left\|u_{i}\right\|_{L^{2}} \leq C=C\left(K,\|Q\|_{L^{2}}\right),\left\|u_{i} / c_{i}\right\|_{L^{\infty}} \leq$ $C=C\left(K,\|Q\|_{L^{2}}\right), i \geq 2$.

Proof. We convert equation (2.5) into an integral equation

$$
\left(^{*}\right)\left\{\begin{array}{l}
u_{1}(x)=1+\int_{-\infty}^{x}\left(\sum_{j \neq 1} Q_{1 j}\right) u_{j}, \\
u_{i}(x)=\int_{-\infty}^{x} e^{c_{i}^{2}(x-y)}\left[c_{i}^{2} Q_{i 1} u_{1}+\sum_{j \neq 1, i} c_{i} Q_{i j} u_{j}\right], \quad i=2,3, \ldots, n .
\end{array}\right.
$$

At first we look for the solution $u_{1} \in L^{\infty}, u_{i} \in L^{2}, i \geq 2$. Let $X$ be the Banach space $\left\{u=\left(u_{1}, u_{2}, u_{3}, \ldots, u_{n}\right): u_{1} \in L^{\infty}, u_{i} \in L^{2}, i \geq 2\right\}$, with the norm $\|u\| \|=\operatorname{Max}_{2 \leq i \leq n}\left\{\left\|u_{1}\right\|_{L^{\infty}},\left\|u_{i}\right\|_{L^{2}}\right\}$.

Case 1. Note that

$$
h_{i}(x)=\left\{\begin{array}{ll}
e^{c_{i}^{2} x}, & x \geq 0 \\
0, & x<0 .
\end{array} \text { is in } L^{1} \cap L^{2},\right.
$$

and $\left\|h_{i}\right\|_{L^{1}}=1 /\left|\operatorname{Re} c_{i}^{2}\right|,\left\|h_{i}\right\|_{L^{2}}=1 / \sqrt{2\left|\operatorname{Re} c_{i}^{2}\right|}$. If $u_{1} \in L^{\infty}, u_{i} \in L^{2}, i \geq 2$, then $Q_{i j} u_{j} \in L^{1}$ for $j \neq 1, Q_{i 1} u_{1} \in L^{2}$, and

$$
\begin{aligned}
\left|\int_{-\infty}^{x}\left(\sum_{j \neq 1} Q_{1 j}\right) u_{j}\right| & \leq \sum_{j \neq 1}\left\|Q_{1 j}\right\|_{L^{2}}\left\|u_{j}\right\|_{L^{2}} \leq\|Q\|_{L^{2}}\|u\| \| ; \\
\left\|h_{i} * c_{i}^{2} Q_{1 l} u_{1}\right\|_{L^{2}} & \leq\left\|h_{i}\right\|_{L^{1}} \mid c_{i}^{2}\left\|Q_{i 1}\right\|_{L^{2}}\left\|u_{1}\right\|_{L^{\infty}} \\
& \leq\left(\left|c_{i}^{2}\right| /\left|\operatorname{Re} c_{i}^{2}\right|\right)\left\|Q_{i 1}\right\|_{L^{2}}\left\|u_{1}\right\|_{L^{\infty}} \\
& \leq K\left\|Q_{i 1}\right\|_{L^{2}}|\|u\||
\end{aligned}
$$


for $j \neq 1, i, Q_{i j} u_{j} \in L_{1}$,

$$
\begin{aligned}
\left\|h_{i} * c_{i} Q_{i j} u_{j}\right\|_{L^{2}} & \leq\left\|h_{i}\right\|_{L^{2}}\left|c_{i}\right|\left\|Q_{i j}\right\|_{L^{2}}\left\|u_{j}\right\|_{L^{2}} \\
& \leq\left(\left|c_{i}\right| / \sqrt{2 \mid \operatorname{Re} c_{i}^{2}} \mid\right)\left\|Q_{i j}\right\|_{L^{2}}\left\|u_{j}\right\|_{L^{2}} \\
& \leq \sqrt{K / 2}\left\|Q_{i j}\right\|_{L^{2}} \mid\|u\| \| .
\end{aligned}
$$

Hence $\left\|h_{i} * c_{i}^{2} Q_{i 1} u_{1}+\sum_{j \neq 1, i} h_{i} * c_{i} Q_{i j} u_{j}\right\|_{L^{2}} \leq(K+\sqrt{K / 2})\|Q\|_{L^{2}}\|u\| \|$. The integral equation $(*)$ is of the form $u=(1,0,0, \ldots, 0)+K u$, where $\|K u\| \mid \leq$ $\alpha\|Q\|_{L^{2}} \mid\|u\| \|$ and $\alpha=\operatorname{Max}\{1, K+\sqrt{K / 2}\}$. If $\|Q\|$ is small enough such that $\alpha\|Q\|<1$, we may solve $u \in X$ and $\|u\| \leq 1 /(1-\alpha\|Q\|)$; i.e., $\left\|u_{1}\right\|_{L^{\infty}} \leq$ $1 /(1-\alpha\|Q\|),\left\|u_{i}\right\|_{L^{2}} \leq 1 /(1-\alpha\|Q\|), i \geq 2$. Recall that

$$
\begin{gathered}
u_{i}(x)=h_{i} * c_{i}^{2} Q_{i 1} u_{1}(x)+\int_{-\infty}^{x} e^{c_{i}^{2}(x-y)}\left(\sum_{j \neq 1, i} c_{i} Q_{i j} u_{j}\right) \quad \text { for } i \geq 2, \\
\left|u_{i}(x)\right| \leq\left\|h_{i}\right\|_{L^{2}}\left|c_{i}^{2}\right|\left\|Q_{i 1}\right\|_{L^{2}}\left\|u_{1}\right\|_{L^{\infty}}+\sum_{j \neq 1, i}\left|c_{i}\right|\left\|Q_{i j}\right\|_{L^{2}}\left\|u_{j}\right\|_{L^{2}} \\
\leq\left(\left|c_{i}^{2}\right| / \sqrt{2\left|\operatorname{Re} c_{i}^{2}\right|}+\left|c_{i}\right|\right)\|Q\|_{L^{2}}|\|u \mid\|, \\
\left|u_{i}(x)\right| \leq\left|c_{i}\right|(\sqrt{K / 2}+1)\|Q\|_{L^{2}}(1 /(1-\alpha\|Q\|)) .
\end{gathered}
$$

Hence

$$
\left\|u_{i} / c_{i}\right\|_{L^{\infty}} \leq(\sqrt{K / 2}+1)\|Q\|_{L^{2}}(1 /(1-\alpha\|Q\|)) \quad \text { for } i \geq 2 .
$$

Case 2. Let $N$ be a positive integer satisfying $\alpha\|Q\|_{L^{2}} / N<1$. There exists a fine number of points $x_{0}=-\infty, x_{1}, x_{2}, \ldots, x_{M}=\infty$ such that $\sum_{i, j}\left(\int_{x_{k}}^{x_{k+1}}\left|Q_{i j}\right|^{2}\right)^{1 / 2} \leq\|Q\|_{L^{2}} / N$.

By Case 1 , the solutions $u_{i}$ exist up to the point $x_{1}$. Then we consider the following equations with initial values at $x_{1}$ :

$$
\left\{\begin{array}{l}
u_{1}(x)=u_{1}\left(x_{1}\right)+\int_{x_{1}}^{x}\left(\sum_{j \neq 1} Q_{1 j}\right) u_{j}, \\
u_{i}(x)=e^{c_{i}^{2}\left(x-x_{1}\right)} u_{i}\left(x_{1}\right)+\int_{x_{1}}^{x} e^{c_{i}^{2}(y-x)}\left[c_{i}^{2} Q_{i 1} u_{1}+\sum_{j \neq 1, i} c_{i} Q_{i j} u_{j}\right], \quad i \geq 2 .
\end{array}\right.
$$

since $\alpha \sum_{i j}\left(\int_{x_{1}}^{x_{2}}\left|Q_{i j}\right|^{2}\right)^{1 / 2}<\alpha\|Q\|_{L^{2}} / N<1$. Again by Case 1 we may extend the solutions $u_{i}$ to the points $x_{2}$. If we continue, we obtain $u_{i}$ defined on the whole line and $\left\|u_{1}\right\|_{L^{\infty}} \leq C=C\left(K,\|Q\|_{L^{2}}\right),\left\|u_{i}\right\|_{L^{2}} \leq C=C\left(K,\|Q\|_{L^{2}}\right)$, $\left\|u_{i} / c_{i}\right\|_{L^{\infty}} \leq C=C\left(K,\|Q\|_{L^{2}}\right)$ for $i \geq 2$. We are done.

Let $m$ be the solution of

$$
\begin{aligned}
& d m / d x=z^{2}[J, m]+(z Q+P) m, \quad Q, P \text { are off-diagonal, } \\
& m(x, z) \rightarrow I \text { as } x \rightarrow-\infty, z \notin \Sigma .
\end{aligned}
$$

For $Q, Q_{x}, P, P_{x} \in L^{1}, m$ is solved in [8,9]. Note that $m(x, z) \rightarrow$ $\exp \left(-\int_{-\infty}^{\infty}\left(Q(\operatorname{ad} J)^{-1} Q\right)^{\mathrm{diag}}\right) \delta(z), \delta(z)=\operatorname{diag}\left(\delta_{1}(z), \delta_{2}(z), \ldots, \delta_{n}(z)\right)$ as $x \rightarrow+\infty$. 
(2.7) Theorem. Let $Q=\left(Q_{i j}\right), P=\left(P_{i j}\right)$ be off-diagional and of Schwartz class. If $|z| \geq 1$ and $\frac{1}{2} \leq|\operatorname{Re} z| /|\operatorname{Im} z| \leq 2$, we may control the solution $m(x, z)$ in $(2.6)$ by the $L^{2}$-norm of $(Q, P)$; i.e., $\left\|m_{i j}(\cdot, z)\right\|_{L^{\infty}} \leq C=$ $C\left(\|Q\|_{L^{2}},\|P\|_{L^{2}},\left|\delta_{1}(z)\right|,\left|\delta_{2}(z)\right|, \ldots,\left|\delta_{n}(z)\right|\right)$.

Proof. It suffices to consider the case $\operatorname{Im} z^{2}>0$. The first column of $m$ satisfies

$$
\left\{\begin{array}{l}
m_{11}=1+\int_{-\infty}^{x} \sum_{j \neq 1}\left(z Q_{1 j}+P_{1 j}\right) m_{j 1}, \\
m_{l 1}=\int_{-\infty}^{x} e^{z^{2}(y-x)\left(i d_{1}-i d_{l}\right)} \sum_{j \neq l}\left(z Q_{l j}+P_{l j}\right) m_{j 1}, \quad l \geq 2 .
\end{array}\right.
$$

Multiplying by $z$ on both sides of the second equation of (2.8), we have

$$
\left\{\begin{array}{l}
m_{11}=1+\int_{-\infty}^{x} \sum_{j \neq 1}\left(Q_{1 j}+P_{1 j} / z\right)\left(z m_{j 1}\right), \\
z m_{l 1}=\int_{-\infty}^{x} e^{z^{2}(y-x)\left(i d_{1}-i d_{l}\right)}\left[\left(z^{2} Q_{l 1}+z P_{l 1}\right) m_{11}+\sum_{j \neq l, 1}\left(z Q_{l j}+P_{l j}\right)\left(z m_{j 1}\right)\right] .
\end{array}\right.
$$

Let $m_{11}=u_{1}, z m_{j 1}=u_{j}, j \geq 2$. By Lemma (2.4), we have $\left\|m_{11}(\cdot, z)\right\|_{L^{\infty}}$ $\leq C\left(\|Q\|_{L^{2}},\|P\|_{L^{2}}\right)$ and $\left\|z m_{j 1}(\cdot, z)\right\|_{L^{\infty}} \leq|z| C\left(\|Q\|_{L^{2}},\|P\|_{L^{2}}\right)$ for $j \geq 2$. Hence $\left\|m_{j 1}(\cdot, z)\right\|_{L^{\infty}} \leq C\left(\|Q\|_{L^{2}},\|P\|_{L^{2}}\right)$ for all $j$. Let $\Lambda^{k}\left(\mathbf{C}^{n}\right)$ denote the space of alternating $k$-forms on $\mathbf{C}^{n}, 1 \leq k \leq n$. It has a standard basis $\left\{e_{j_{1}} \wedge e_{j_{2}} \wedge \cdots \wedge e_{j_{k}}: 1 \leq j_{1}<j_{2}<\cdots<j_{k} \leq n\right\}$. The wedge product $f_{k}(\cdot, z)=$ $m_{1}(\cdot, z) \wedge m_{2}(\cdot, z) \wedge \cdots \wedge m_{k}(\cdot, z)$ is a $\Lambda^{k}\left(\mathbf{C}^{n}\right)$-valued function and satisfies the differential equation:

$$
\begin{aligned}
\frac{\partial f_{k}}{\partial k}= & z^{2} \sum_{j=1}^{k} m_{1} \wedge m_{2} \wedge \cdots \wedge m_{j-1} \wedge J m_{j} \wedge m_{j+1} \wedge \cdots \wedge m_{k} \\
& -z^{2}\left(i d_{1}+i d_{2}+\cdots+i d_{k}\right) f_{k} \\
& +\sum_{j=1}^{k} m_{1} \wedge m_{2} \wedge \cdots \wedge m_{j-1} \wedge(z Q+P) m_{j} \wedge m_{j+1} \wedge \cdots \wedge m_{k} .
\end{aligned}
$$

Let

$$
\begin{gathered}
N=C\left(\begin{array}{l}
n \\
k
\end{array}\right)=n ! /(k !(n-k) !), \\
\left\{C_{1}=0, C_{2}, C_{3}, \ldots, C_{N}\right\} \\
=\left\{i\left(d_{j_{1}}+d_{j_{2}}+\cdots+d_{j_{k}}-d_{1}-d_{2}-\cdots-d_{k}\right): 1 \leq j_{1}<j_{2}<\cdots<j_{k} \leq n\right\}, \\
m_{1} \wedge m_{2} \wedge \cdots \wedge m_{k}=\sum_{j_{1}<j_{2}<\cdots<j_{k}} u_{\sigma_{\left\{j_{1}, j_{2}, \ldots, j_{k}\right\}} e_{j_{1}} \wedge e_{j_{2}} \wedge \cdots \wedge e_{j_{k}} .}
\end{gathered}
$$

Here $\sigma$ is a one-one correspondence from $\left\{\left(j_{1}, j_{2}, \ldots, j_{k}\right), 1 \leq j_{1}<j_{2}<\cdots<\right.$ $\left.j_{k} \leq n\right\}$ to $\{1,2, \ldots, N\}$ with $\sigma(1,2,3, \ldots, k)=1$. Then $u_{i}$ satisfies

$$
\frac{d u_{i}}{d x}=z^{2} C_{i} u_{i}+\sum_{j \neq i}\left(z \widetilde{Q}_{i j}+\widetilde{P}_{i j}\right) u_{j} \text {. }
$$


Let

$$
\begin{gathered}
i=\sigma\left(i_{1}, i_{2}, \ldots, i_{k}\right), \quad \alpha \notin\left\{i_{1}, i_{2}, \ldots, i_{k}\right\}, \\
l=\sigma\left(i_{1}, i_{2}, \ldots, i_{j-1}, \alpha, i_{j}, \ldots, i_{k}\right) .
\end{gathered}
$$

Then $\widetilde{Q}_{i l}=Q_{i_{j} \alpha}, \widetilde{P}_{i l}=P_{i_{j} \alpha}, \widetilde{Q}_{i j}=\widetilde{P}_{i j}=0$ otherwise. Hence $\|\widetilde{Q}\|_{L^{2}} \leq\|Q\|_{L^{2}}$, $\|\widetilde{P}\|_{L^{2}} \leq\|P\|_{L^{2}} . \quad C_{\sigma\left\{j_{1}, j_{2}, \ldots, j_{k}\right\}}=i\left(d_{j_{1}}+d_{j_{2}}+\cdots+d_{j_{k}}-d_{1}-d_{2}-\cdots-d_{k}\right)$, $j_{1}<j_{2}<\cdots<j_{k}, C_{1}=0, \operatorname{Im} C_{j}>0$ for $j \geq 2$. We have the same type of equation as the first column of $m$. Hence $\left\|u_{i}(\cdot, z)\right\|_{L^{\infty}} \leq C\left(\|Q\|_{L^{2}},\|P\|_{L^{2}}\right)$ for $|z| \geq 1$. Note that

$m \rightarrow \exp \left(-\int_{-\infty}^{\infty}\left(Q(\operatorname{ad} J)^{-1} Q\right)^{\operatorname{diag}}\right) \delta(z), \quad \delta(z)=\operatorname{diag}\left(\delta_{1}(z), \delta_{2}(z), \ldots, \delta_{n}(z)\right)$

as $x \rightarrow \infty$, and we have Volterra equations for $m_{n}, m_{n-1} \wedge m_{n}, m_{n-2} \wedge m_{n-1} \wedge$ $m_{n}, \ldots$ normalized at $x=\infty$. Since $\operatorname{det} m=1$,

$$
\begin{gathered}
\left\|m_{i}(x, z)\right\| \leq\left\|m_{1}(x, z) \wedge m_{2}(x, z) \wedge \cdots \wedge m_{i}(x, z)\right\| \\
\cdot\left\|m_{i}(x, z) \wedge m_{i+1}(x, z) \wedge \cdots \wedge m_{n}(x, z)\right\| .
\end{gathered}
$$

Finally, we have $\left\|m_{i j}(\cdot, z)\right\|_{L^{\infty}} \leq C\left(\|Q\|_{L^{2}},\|P\|_{L^{2}},\left|\delta_{1}(z)\right|,\left|\delta_{2}(z)\right|, \ldots,\left|\delta_{n}(z)\right|\right)$ for $|z| \geq 1$.

(2.11) Remark. The existence of $m$ in (2.6) was proved in [8,9]. The point of Theorem (2.7) is to control $m(x, z)$ by the $L^{2}$-norm of $(Q, P)$ for $z$ large.

\section{Evolution EQUATIONS}

Suppose the scattering data $v(z, t)$ evolves as

$$
\begin{cases}d v(z, t) / d t=z^{k-1}[J, v(z, t)], & \\ d v\left(z_{j}, t\right) / d t=z_{j}^{k-1}\left[J, v\left(z_{j}, t\right)\right], & k \text { is an odd positive integer, } \\ & z_{j} \text { is fixed for each } j .\end{cases}
$$

Assume $v(z, 0) \in \mathrm{SD}$ is generic. Then $v(z, t)$ is generic for $t$ small (since the set of the generic potentials is open in SD) $[2,8,10]$. The corresponding potential $(Q(x, t), P(x, t))$ satisfies the following evolution equation:

$$
\left\{\begin{array}{l}
Q_{t}=\left[J, F_{k}\right] \\
P_{t}=\left[Q, F_{k}\right]+\left[J, F_{k+1}\right],
\end{array}\right.
$$

where $F_{k}=F_{k}(Q, P)$ are computed by the recurrence formula

$$
d F_{k} / d x-\left[P, F_{k}\right]=\left[Q, F_{k+1}\right]+\left[J, F_{k+2}\right], \quad F_{0}=J \quad[8,10,12] .
$$

Let $q=(Q, P)$; equation (3.2) is of the form $q_{t}(\cdot, t)=F(q(t, \cdot))$.

(3.3) Definition. We say that the evolution is dissipative if $\operatorname{Re} \int \operatorname{tr}\left[q^{*} F(q) d x\right]$ $\leq 0$ for all $q$. 
Let $\|a\|=\operatorname{tr}\left(a^{*} a\right)^{1 / 2}$ be a norm of $M_{n}(\mathbf{C}) .\|q\|_{2}^{2}=\int \operatorname{tr}\left(q^{*}(x, t) q(x, t)\right) d x$. (Note that this norm is equivalent to $\|q\|_{L^{2}}=\sum_{i j}\left(\int\left|q_{i j}\right|^{2} d x\right)^{1 / 2}$.)

$$
\frac{d}{d t} \int \operatorname{tr}\left(q^{*} q\right) d x=2 \operatorname{Re} \int \operatorname{tr}\left(q^{*} q_{t}\right) d x .
$$

Therefore if $q(x, t)$ is the solution of a dissipative evolution equation, then $\|q(\cdot, t)\|_{2} \leq c\|q(\cdot, 0)\|_{2}$.

(3.4) Theorem. If $(Q(x, 0), P(x, 0))=\left(Q_{0}, P_{0}\right)$ is a generic potential of Schwartz class and the evolution equation (3.1) is "dissipative", then $(Q(x, t)$, $P(x, t))$ exists for all $t \in R$.

Proof. Let $m$ be the eigenfunction associated with $(Q, P)$. Note that $m \rightarrow$ $\delta(z)=\operatorname{diag}\left(\delta_{1}(z), \delta_{2}(z), \ldots, \delta_{n}(z)\right)$ as $x \rightarrow \infty, \delta(z)$ is invariant under the evolution (3.1) [8, 10], and $\delta(z) \rightarrow I$ as $|z| \rightarrow \infty$. By Theorem (2.7), $\left\|m_{i j}(\cdot, t, z)\right\|_{L^{\infty}} \leq C\left(\left\|Q_{0}\right\|_{L^{2}},\left\|P_{0}\right\|_{L^{2}}\right)$ for $z$ large.

$$
m(x, t, \cdot)=r(x, t, \cdot) m^{\#}(x, t, \cdot) \exp \left(x z^{2} J\right) u(x, t, \cdot) \exp \left(-x z^{2} J\right) .
$$

Let $\operatorname{sd}\left(Q_{0}, P_{0}\right)=v(z, 0)$. Since the generic scattering data is open in $\mathrm{SD}$, there exists $T>0, v(z, t)$ is generic for $t<T$. By the same argument as in [3, 11],

$$
\begin{aligned}
& \left\|r\left(x, t_{\nu}, z\right)\right\| \leq c \quad \text { for } x \in R, \quad \frac{1}{2} \leq|\operatorname{Im} z| /|\operatorname{Re} z| \leq 2, \\
& z \text { large }, t_{\nu}<T, t_{\nu} \rightarrow T .
\end{aligned}
$$

Since $\left\|m_{i j}(\cdot, t, z)\right\|_{L^{\infty}} \leq C\left(\left\|Q_{0}\right\|_{L^{2}},\left\|P_{0}\right\|_{L^{2}}\right),\left\|r\left(x, t_{\nu}, z\right)\right\| \leq C$ for $t_{\nu}<T$, $z$ large. Passing to a subsequence, we deduce that $r\left(x, t_{\nu}, \cdot\right) \rightarrow r(x, T, \cdot)$, where the residues of $r(x, T, \cdot)$ solve the requisite linear equation at $t=T$. Therefore $v(\cdot, T)$ is generic. Since the set of of generic data is open in SD, the solution $(Q(x, t), P(x, t))$ of equation (3.2) exists for $0 \leq t<T+\varepsilon$. Obviously this implies the global existence of equation (3.1).

\section{EXAMPLES}

By a slight modification the argument in $\S 3$ works for the case

$$
J=\operatorname{diag}(-N i, i, i, \ldots, i), \quad Q=\left(\begin{array}{ccccc}
0 & q_{1} & q_{2} & \cdots & q_{n} \\
r_{1} & & & & \\
r_{2} & & & \\
\vdots & & & \\
r_{n} & & &
\end{array}\right), \quad r_{i}= \pm q_{i}^{*} .
$$

The associated evolution is an $N$-component derivative nonlinear Schrödinger equation:

$$
\left(q_{j}\right)_{l}=i\left(q_{j}\right)_{x x}+\varepsilon \alpha\left(\sum_{k}\left(q_{k} q_{k}^{*}\right) q_{j}\right)_{x}, \quad \varepsilon= \pm 1, j=1,2, \ldots, n .
$$


Since $\frac{d}{d t} \int \sum_{j}\left(q_{j}^{*} q_{j}\right)=0$, the $L^{2}$-norm of $Q$ is invariant under the evolution (4.2). We have global existence for the $N$-component derivative nonlinear Schrödinger equation if $Q(x, 0)$ is generic and of Schwartz class. For $n=1,(4.1)$ becomes the derivative nonlinear Schrödinger equation (DNLS). The global existence of DNLS was obtained in [11]. Let $\psi=m \exp \left(x z^{2} J\right)$; then (4.1) becomes

$$
d \psi / d x=\left(z^{2} J+z Q\right) \psi .
$$

For $n=2$ Kaup and Newell obtained soliton solutions for the spectral problem (4.3) [7]. Gerzhikov et al. also considered this case [6]. Morris and Dodd considered the two-component derivative nonlinear Schrödinger equation using a larger scattering problem (i.e., $n=3$ ) [15]. Sasaki derived a Hamiltonian structure for the evolution (4.2) [16].

Acknowledgement. The comments of the referee leading to the present version are deeply appreciated. This work was partially supported by the National Science Council of the R.O.C.

\section{REFERENCES}

1. R. Beals and R. R. Coifman, Scattering transformation spectrales, et équations d'évolution non linéaires, Séminaire Goulaouic-Meyer-Schwartz 1980-1981, exp. 22, École Polytechnique, Palaiseau.

2. __ Scattering and inverse scattering for first order system, Comm. Pure Appl. Math. 37 (1984), 39-90.

3. __ Inverse scattering and evolution equations, Comm. Pure Appl. Math. 38 (1985), 29-42.

4. __ Scattering and inverse scattering for first-order system: II. Inverse problem 3 (1987), 577-593.

5. R. K. Bullough and P. J. Caudrey (eds.), Solitons, Topics Current Phys., no. 17, SpringerVerlag, New York, 1980.

6. V. S. Gerezhikov et al., Quadratic bundle and nonlinear equations, Theoret. and Math. Phys. 44 (3) (1980), 784-795.

7. D. J. Kaup and A. C. Newell, An exact solution for a derivative nonlinear Schrödinger equation, J. Math. Phys. 19 (4) (1978), 789-801.

8. J. H. Lee, Analytic properties of Zakharov-Shabat inverse scattering problem with polynomial spectral dependence of degree 1 in the potential, Ph.D. Dissertation, Yale Univ., May 1983.

9. __ A Zakharov-Shabat inverse scattering problem and the associated evolution equations, Chinese J. Math. (4) (1984), 223-233.

10. __ Analytic properties of a Zakharov-Shabat inverse scattering problem (I), Chinese J. Math. (4) (1986), 225-248.

11. __ Global solvability of the derivative nonlinear Schrödinger equation, Trans. Amer. Math. Soc. (to appear).

12. __ Analytic properties of a Zakharov-Shabat inverse scattering problem (II), preprint.

13. W. Mio et al., Modified nonlinear Schrödinger equation for Alfvén waves propagating along the magnetic field in cold plasmas, J. Phys. Soc. Japan 41 (1976), 265-271.

14. E. Mjølhus, On the modulational instability of hydromagnetic waves parallel to the magnetic field, J. Plasma Phys. 16 (1976), 321-334. 
15. H. C. Morris and R. K. Dodd, The two component derivative nonlinear Schrödinger equation, Phys. Scripta 20 (1979), 505-508.

16. R. Sasaki, Canonical structure of soliton equations, II. The Kaup-Newell system, Physica 5D (1982), 66-74.

17. V. E. Zakharov and A. B. Shabat, A refined theory of two-dimensional self-focussing and onedimensional self-modulation of waves in non-linear media, Soviet Phys. JETP 34 (1972), 62-69.

Institute of Mathematics, Academic Sinica, Taipei 11529, Taiwan, R.O.C. 